\title{
LA EDUCACIÓN FÍSICA Y EL DEPORTE COMO MEDIOS DE TRANSGRESIÓN DEL MODELO DE MUJER DURANTE LA DICTADURA FRANQUISTA
}

\author{
A EDUCAÇÃO FÍSICA E O ESPORTE COMO MEIOS DE TRANSGRESSÃO DO \\ MODELO DE MULHER DURANTE A DITADURA FRANQUISTA
}

PHYSICAL EDUCATION AND FEMALE SPORTS AS WAYS OF TRANSGRESSING THE FEMALE MODEL DURING FRANCO'S DICTATORSHIP

Gonzalo Ramírez-Macías*

\section{Palabras clave:}

Mujeres.

Educación y

Entrenamiento

físico.

Deportes.

España.

Palavras-chave:

Mulheres.

Educação e

Treinamento físico.

Desportos.

Espanha.

\section{Keywords:}

Women.

Physical Education

and Training.

Sports.

Spain
Resumen: Durante la dictadura franquista (1939-1975) la Educación Física y el deporte femenino fueron originariamente definidos como instrumentos para la transmisión del ideal de mujer que se trató de imponer. El objetivo de este artículo es dilucidar si estas prácticas evolucionaron y llegaron a ser medios de transgresión del modelo femenino impuesto. Para ello se han utilizado fuentes primarias y aportaciones relevantes de fuentes secundarias. Las conclusiones muestran que tanto la Educación Física como el deporte femenino fueron medios de transgresión del modelo femenino impuesto, si bien no llegaron a oponerse de forma directa a dicho modelo.

Resumo: Durante a ditadura de Franco (1939-1975) a Educação Física e o esporte feminino definiram-se como instrumentos para a transmissão da mulher ideal. $O$ objetivo principal deste estudo é elucidar se estas atividades converteram-se em formas de transgressão do modelo feminino. A investigação utilizou fontesprimárias e fontes secundárias relevantes. Os resultados mostraram que a tanto a Educação Física quanto o esporte foram mecanismos de transgressão do modelo feminino, mas não se opuseram diretamente a esse modelo.

Abstract: During Franco's dictatorship (1939-1975), Physical Education and female sports were defined as instruments for conveying the female ideal. The main objective of this paper is to know if those activities became ways of transgressing the female model imposed. The investigation used primary sources and relevant secondary sources. The findings show that Physical Education and female sports were ways of transgressing the female model imposed, but they did not oppose that model directly..
*Universidad de Sevilla. Sevilla, España.

E-mail: grm@us.es

Recebido em: 07-09-2016 Aprovado em: 20-02-2018

DOI: http://dx.doi.org/10.22456/1982-8918.78334 (c) (1) (3) Licence 


\section{INTRODUCCIÓN}

La dictadura franquista (1939-1975) significó un importante freno al desarrollo de las mujeres que se había ido forjando en el primer tercio de siglo XX y en especial durante la II República. Para la imposición de su modelo femenino, la cúpula de poder franquista, formada por el Movimiento Nacional', el Ejército y la Iglesia Católica, no escatimó en medios: médicos, maestros, políticos, sacerdotes,... urdieron un arquetipo de mujer que quedaba restringido a los roles de ama de casa, esposa y madre, asociando de forma indisoluble los términos mujer y hogar para la construcción de la feminidad; a estos roles se les sumaban los de feligresa católica y patriota (ALTED, 1991).

No obstante, en unaetapa que duró casi cuarenta años, este concepto de mujer no se mantuvo inalterable.A partir de la década de 1960 España experimentó un necesario aperturismo a nivel internacional, que permitió un progresivo avance económico, pero que también comenzó a dilapidar la estructura social propugnada durante el periodo anterior.Todo ello produjo cambios en torno a la concepción de la mujer; no obstante Manrique (2003) y Alted (1991) defienden que ello no implicó que los aspectos definitorios del modelo de mujer se modificaran, sino que se le añadieron nuevos matices, los cuales fueron de carácter progresista.

Uno de los medios que utilizó el régimen para la transmisión de su modelo femenino fue la Educación Física y el deporte, que estuvieron en manos de la Sección Femenina². Estudios precedentes como los de Ramírez-Macías (2012), Manrique (2003) o Zagalaz (2001) inciden en diferentes aspectos de la estructura y funcionamiento de la Educación Física y el deporte femenino durante este periodo. Sin embargo, hay que destacar que todos ellos, si bien de soslayo, abren el interrogante de si la Educación Física y el deporte también sirvieron como válvula de escape a las restricciones marcadas por el arquetipo femenino. En torno a este asunto gira esta investigación, planteándose si la Educación Física y el deporte fueron medios delos que se sirvieron las mujeres de la época para transgredir los rígidos límites de ama de casa, esposa y madre.En caso de que esta cuestión se responda afirmativamente, cabe preguntarse cómo la Educación Física y el deporte se implementaron como medios no sólo para transgredir el rígido arquetipo femenino, sino para añadirlenuevosmatices progresistas. Es decir, de forma más concreta, ¿cuáles fueron esas transgresiones del modelo femenino franquista a partir de la Educación Física y el deporte?, ¿determinaron dichas transgresiones la asimilación,por parte de las mujeres, de nuevos roles progresistas?

Esta investigación tratará de responder a estas preguntas, para lo cual se fundamentará en el método de investigación histórico, a partir de una orientación hermenéutica-interpretativa. Se trata de una metodología consolidada en las ciencias sociales (FLICK, 2007), que trata de responder a las cuestiones planteadas a partir de un análisis hermenéutico de las fuentes. Más específicamente, la técnica utilizada para el análisis de dichas fuentes es la revisión documental (MARSHALL; ROSSMAN, 2011), la cual se basa en la recogida y análisis de información relevante para responder a las preguntas formuladas en la investigación. En concreto, las fuentes utilizadas en este estudio son principalmente primarias. En primer lugar

1 Mediante el Decreto de 19 de abril de 1937 Franco incorporó a todos los sectores que le apoyaban (principalmente monárquicos, tanto carlistas como alfonsinos, falangistas y corporativistas católicos) al llamado Movimiento Nacional, organismo que dependía directamente de su persona. 2 La Sección Femenina fue fundada en 1934, constituyendo la rama femenina de la Falange Española Tradicionalista. El Decreto de 28 de diciembre de 1939 le encomendaba las siguientes funciones: Movilización, encuadramiento y formación de las afiliadas; formación política y educación profesional de las mujeres encuadradas en las restantes Secciones del Movimiento; formación para el hogar y Servicio Social de la mujer. A ello hay que sumar que la Ley de 6 de diciembre que le confió la docencia de la Educación Física de todas sus afiliadas y del resto de la juventud no afiliada, es decir, la juventud femenina en su conjunto. 
la documentación contenida en el Fondo de la Asociación Nueva Andadura, series Azul y Roja. En segundo lugarla documentación legislativa obtenida del Boletín Oficial del Estado. En tercer lugar hay que subrayar la importancia que han tenido para este estudio las publicaciones oficiales de la Sección Femenina, así como los discursos, entrevistas, memorias y publicaciones oficiales de personajes relevantes dentro de esta organización, como Pilar Primo de Rivera 0 María de Miranda. En cuarto lugar señalar que se han utilizado, si bien de forma puntual, fuentes hemerográficas. Todo ello se ha completado con fuentes secundarias, especialmente de estudios precedentes relacionados con éste, que han completado los datos que se han obtenido en las fuentes primarias.

\section{EL MODELO DE MUJER DURANTE EL FRANQUISMO}

La exposición de las características definitorias del modelo de mujer que durante la dictadura se trató de imponer, permitirá comprender hasta qué punto la Educación Física y el deporte femenino fueron medios de transgresión de dicho modelo. Varios son los estudios precedentes que han analizado el arquetipo femenino durante la dictadura (SCANLON, 1977; MÒDOL, 1991; OFER, 2009) no obstante, es posible encontrar una definición clara al respecto en una obra muy anterior, La perfecta casada de Fray Luis de León ([1583] 1987, p. 34): "[...] la mujer sujeta a la autoridad del esposo reforzaba en el contexto familiar los valores de sumisión, respeto a la autoridad paterna,[...] en definitiva todos aquellos valores que ayudaban a la conservación del orden de las cosas establecidas". En relación a ello Scanlon (1977) y Mòdol (1991) subrayan el valor de la sumisión y la definición de tres roles: hija, esposa y madre. Al gran valor de la sumisión, Ofer (2009) añade compasión, abnegación y disciplina.

Este modelo de mujer venía claramente marcado desde los estamentos rectores de la dictadura, que mediante argumentos religiosos y biológicos trató de justificar la veracidad de su planteamiento. Como ejemplo ilustrativo se presentan las palabras del Pontífice Pio XII en 1945:

\footnotetext{
Su oficio, su manera, su inclinación innata es la maternidad. Toda mujer está destinada a ser madre (en sentido físico o espiritual). A este fin ha ordenado el Creador todo el ser propio de la mujer [...] Y así la mujer, verdaderamente tal, no puede no ver ni entender a fondo todos los problemas de la vida humana más que bajo el aspecto de la familia (PIO XII, 1967, p.1688-1689).
}

Por tanto, las funciones de las mujeres se circunscribían, en la mayoría de los casos, a dos concretas: ser esposa y madre, siempre en el mismo contexto, el hogar. Esta situación incluía un total sometimiento de las mujeres al hombre cabeza de familia; de tal forma que desde el nacimiento hasta el casamiento dependían del padre y, después de éste, dependían para siempre de su marido.

Estas funciones asignadas a las mujeres conectaban directamente con lo que Campos (2016) denomina la eugenesia punitiva franquista, auspiciada en gran medida por VallejoNájera y que propugnaba una eugenesia de corte ambientalista, que buscaba mejorar la raza española extirpando del cuerpo social a los enemigos políticos del país, buscando la moralización de las nuevas generaciones en base al nacional-catolicismo franquista (ÁLVAREZ, 1997). Dentro de esta política de supuesta regeneración racial, el papel de las mujeres era esencial, pues eran el elemento clave en la transmisión de esa ideología a sus hijos e hijas y las encargadas de mantener esos principiosmoralizantes en un contexto específico: el hogar (CAMPOS;HUERTAS, 2012). 
En cuanto a las posibilidades de que las mujeres trabajaran o estudiaran, se consideraba que eran situaciones especiales que salían de lo impuesto, tolerándose sólo en el caso de que estuvieran sujetas a condiciones muy estrictas, adecuadas a su género. Para justificar esta posición, los dirigentes franquistas se apoyaban en unas teorías que pretendían ser científicas, a partir de los escritos de médicos reputados como Gregorio Marañón o Antonio De la Granda. Este último escribió:

El duro e insoslayable yugo sexual de la mujer (menstruación, embarazo, parto, lactancia y menopausia) determinan su cuerpo y su mente [...], de forma que está saturada de feminidad y maternidad lo que impide el vuelo libre de sus facultades mentales (DE LA GRANDA, 1947, p. 492).

Al respecto, es imprescindible citar a la Delegada Nacional de la Sección Femenina, Pilar Primo de Rivera:

Las mujeres nunca descubren nada: les falta, desde luego, el talento creador reservado por Dios para inteligencias varoniles: nosotras no podemos hacer más que interpretar mejor o peor lo que los hombres nos dan hecho (PRIMO DE RIVERA, 1942, p. 22).

La base principal de los Estados es la familia y, por tanto, el fin natural de todas las mujeres es el del matrimonio. Por eso la Sección Femenina tiene que prepararlas para cuando llegue para ellas ese día, sepan decorosamente dirigir su casa y educar a sus hijos (PRIMO DE RIVERA, 1942, p. 88).

Por tanto, encontramos un estereotipo del género femenino, justificado a partir de supuestos argumentos científicos y religiosos, inhibidor y muy represivo para las mujeres, marcado, defendido e impuesto severamente desde los órganos de poder del régimen y que se caracterizaba por: sumisión, pasividad, impresionabilidad, abnegación, fragilidad e inferioridad intelectual (PASTOR, 1984). Este estereotipo formaba parte de lo que Herrero (2002) ha llamado el imaginario social del franquismo, definido como la conformación de las representaciones colectivas que rigieron este orden social (ilegítimo en su origen) y que llegó a ser considerado como algo natural 0 , al menos, prácticamente incontestado. Al respecto, es importante reseñar que aquellas mujeres que no se atenían a este ideal eran vilipendiadas y desdeñadas. De hecho, Núñez (2004, p. 47) afirma que estas mujeres servían como ejemplo del "infierno terrenal en el que se podía caer". Entre ellas no sólo estaban las mujeres solteras o las homosexuales, sino que destacaban especialmente tres modelos no integrados en el franquismo: presas políticas, familiares de presos políticos y prostitutas (NÚÑEZ, 2004).

No obstante, como ya se indicó en la introducción, en un periodo que duró casi cuarenta años, el modelo de mujer imperante durante la dictadura no se mantuvo inalterable. El llamado Desarrollismo ${ }^{3}$ trajo nuevos aires, que permitieron a las mujeres españolas liberarse un tanto del encorsetamiento social al que estaban sometidas. Por supuesto no abandonaron la esencia de su rol en la sociedad, pero sumaron nuevos matices al mismo que le permitieron una mayor libertad. Más concretamente, como consecuencia de los cambios en favor de una industrialización de la economía, se generó un gran éxodo hacia las ciudades yuna gran necesidad de mano de obra, lo cual no sóloconllevó la necesaria incorporación de las mujeres a las fábricas, sino una mayor facilidad de acceso a la educación y, por supuesto, la concienciación sobre la importancia de que las niñas recibieran una educación de cara a su futuro, incluso a nivel universitario.

3 A finales de la década de 1950 comenzó un proceso de apertura de España hacia el exterior, así como una política de industrialización y modernización. Todo ello repercutió positivamente en la sociedad española. A esta etapa, historiadores como Preston (2015), la denomina Desarrollismo. 
Estos planteamientos eran impensables en los primeros años de la dictadura. Así, el necesario aperturismo que sufrió España influyó notablemente en la concepción y roles de la mujer, lo cual incluso se reflejó en la legislación; siendo el ejemplo más palpable de ello la Ley 56/1961, de 22 de julio, sobre derechos políticos profesionales y de trabajo de la mujer, que estableció la igualdad de sexos en el trabajo y en el salario, si bien con notables excepciones, como el acceso a la abogacía por parte de las mujeres, posibilidad que no se abriría hasta 1966 (MOLINERO, 1996).

Al respecto hay que subrayar que muchos de estos cambios fueron auspiciados por la propia cúpula de la Sección Femenina, en algunos casos incluso antes dela década de 1960, lo que les llevó en más de una ocasión a caer en verdaderas incongruencias. Como defiende Ofer (2009), es muy desconcertante la defensa que esta organización hizo del modelo de madre y esposa y, a su vez, la defensa de la formación física e intelectual de las mujeres, de sus derechos laborales o de su papel en la política del país.

A pesar de todo lo expuesto,sería un error pensar que la Sección Femenina mantuvo un enfrentamiento directo con el resto de grupos de poder de la dictadura. Realmente, las dirigentes de esta organización nunca se plantearon luchar contra el sistema impuesto, sino que fueron tremendamente hábiles a la hora de localizar lagunas, espacios sin definición dentro de la sociedad, y, por supuesto, a la hora de aprovechar impulsos externos, como lo fue el Desarrollismo, para ir añadiendo y consolidando matices progresistas dentro de los roles asignados a las mujeres. Si bien, es preciso recalcar que los aspectos más conservadores y definitorios del modelo femenino no desaparecieron de su discurso.

\section{EDUCACIÓN FÍSICA Y DEPORTE FEMENINO COMO MEDIOS DE TRANSGRESIÓN DEL MODELO DE MUJER FRANQUISTA}

La Educación Física y el deporte femenino fueron inicialmente un auténtico escándalo para la Iglesia Católica. Por ejemplo el Arzobispo de Valladolid en los años cuarenta llegó a prohibir a las mujeres que montaran en bicicleta por considerarlo pecaminoso (ALTED, 1991) y el Cardenal Segura prohibió la gimnasia en los colegios femeninos de Sevilla, norma que mantuvo hasta su muerte en 1957 (BARRACHINA, 1991).

Este contexto adverso, sobre todo durante la posguerra y la década de 1940, obligó a la cúpula de la Sección Femenina a esforzarse en justificar la importancia y la validez, dentro del modelo impuesto, de las prácticas deportivas para las mujeres. De hecho, María de Miranda (Regidora Central de Educación Física) en su memorándum titulado Sobre la necesidad de Educación Física defendía:

Dándonos este maravilloso cuerpo, Dios nos provee a nosotras de un inconmensurable regalo. Abandonando este regalo, no cuidándolo cometemos una gran ingratitud. Y la misión para la que nosotras hemos sido llamadas no es solamente moral, por tanto estamos obligadas por nuestro deber cristiano a cuidar de él, siendo la Educación Física y el deporte un medio para tal fin (MIRANDA, 1940, documento 49).

Indudablemente es un argumento bastante acrobático para justificar la necesidad de la Educación Física y el deporte, pero no fue el único que presentó de esta índole. Un poco después, aprovechando la posición de la Iglesia Católica en torno al suicidio, afirmó que 
ello implicaba "la necesidad de vigorizar y mejorar nuestra salud física" (MIRANDA, 1942, documento 52). De tal manera que, según ella, la participación en actividades deportivas era equivalente a una obligación moral, pues mejoraban la salud y desterraban ideas que, como el suicidio, eran contrarias al Cristianismo.

En esa misma línea argumentativa, en la que se asocia formación moral, Educación Física y deporte, se expresaba Pilar Primo de Rivera:

Nuestra principal preocupación fue y es que nuestra Educación Física tenga como base un fondo espiritual [...] He aquí la meta de nuestra Educación Física: el perfeccionamiento del cuerpo, a fin de que pueda mejor servir los intereses del alma que en él se encierra (PRIMO DE RIVERA, 1952, p. 87-88).

La Educación Física, que no cabe duda que tiene sus peligros, tiene también inmensas ventajas, como son la disciplina colectiva, la unidad cuando hacen ejercicios juntas varias provincias, la afición al aire y al sol, que las quita del ambiente impuro de los cines y de los bares (PRIMO DE RIVERA, 1942, p. 46).

Afortunadamente estos esfuerzos argumentativos, en pro de la Educación Física y el deporte femenino, lograron que estas prácticas fueran siendo progresivamente aceptadas por la cúpula de poder del régimen, si bien, para que ello fuera posible, fue necesario que tuvieran una relación directa con los roles femeninos establecidos. La justificación de una Educación Física y unas prácticas deportivas específicas para las mujeres, se argumentó, como muy bien ha expuesto Manrique (2003), a partir de explicaciones que provenían de diferentes ámbitos: científico-médico, religioso y político.

En primer lugar, los argumentos científico-médicos determinaron la necesidad de establecer un programa de higiene y salud corporal relacionado directamente con la política de eugenesia ambiental propugnada, es decir, la Educación Física comenzó a tener importancia en relación a la maternidad, mujeres fuertes que dieran a luz a hijos e hijas fuertes, todos ellos educados en los principios del nacional-catolicismo franquista. Hay que destacar al respecto la prohibición expresa de determinados deportes, como el atletismo, excluido hasta 1961 por el excesivo esfuerzo físico que requería y por considerarlo masculinizante (ZAGALAZ, 2001).

En segundo lugar, se hallan los argumentos basados en el catolicismo integrista propio de la época, que marcaba que toda actividad física de las mujeres debía cumplir a rajatabla con las reglas morales ultraconservadoras que impulsó la Iglesia Católica. Ello incluía desde la forma de vestir al comportamiento decoroso, pasando por el hecho de que las prácticas deportivas no debían ser contrarias a las buenas costumbres cristianas.

En tercer lugar se encuentra la política neotradicional, que desde un principio tasó a la Educación Física y al deporte desde un prisma de servicio a la Nación, fundamentado en su potencial adoctrinador (CHAVES, 1960). Así, tanto la Educación Física como el deporte se orientaron como una preparación física para engrandecer España: las mujeresdebían estar listas para servir a España, pero en su ámbito específico que no era otro que el consabido hogar.

A pesar de estos planteamientos iniciales tan rígidos, las mujeres encontraron en la Educación Física y el deporte una vía de liberación del encorsetamiento al que socialmente estaban sometidas. Por supuesto sin un enfrentamiento directo con los poderes rectores del régimen, sino aprovechando las oportunidades que le brindaban las escasas lagunas que planteaba el férreo control existente en torno a la Educación Física y el deporte; porque 
aunque la Iglesia Católica y las facciones más conservadoras del Movimiento Nacional habían transigido en los términos especificados anteriormente, ni mucho menos dejaron de mantener una estrecha vigilancia al respecto. Así, las deportistas tuvieron que enfrentarse a una constante presión que buscaba marcar con claridad los objetivos y límites de estas prácticas. Esta línea no sólo fue sostenida por la Iglesia Católica y los políticos ultraconservadores, ya que hasta los medios de comunicación la defendieron. Sirva como ejemplo este artículo rescatado del Correo de Mallorca (1941, p. 28):

Toda educación femenina ha de ordenarse a la misión que la mujer ha traído al mundo: la maternidad. En lo físico, a la madre le hace falta un estado general de salud y desarrollo corporal bueno, sin que le sea preciso poseer mucho músculo ni fuertes bíceps. La mujer atleta, la campeón[...], no puede ser el ideal de esposa para ningún hombre, e indudablemente, en general, nunca será la mejor madre.

Este tipo de afirmaciones no eran gratuitas y la línea de presión de la que partían tampoco, las mujeres españolas comenzaban a practicar deportes, entrenaban, competían, ganaban y perdían, en definitiva disfrutaban de la misma esencia agonística del deporte. Bien es cierto que, como afirman Pujadas et al. (2014), dentro de la geografía española existieron zonas con diferente nivel de desarrollo. Mientras que el sur ruralizado mantenía una Educación Física y un deporte femenino dominado en gran medida por la Sección Femenina, en los grandes orbes, como Barcelona o Bilbao, las prácticas deportivas femeninas comenzaron a independizarse del dominio institucional, gracias al creciente desarrollo de un tejido asociativo independiente.

En cualquier caso, aunque a diferentes niveles, se estaban rebasando los límites en los que la Educación Física y el deporte eran tolerados, pues valores propios de las mujeres, como la sumisión, la fragilidad y la supuesta inferioridad física, quedaban indudablemente superados dentro del ámbito deportivo. De hecho la mismísima Pilar Primo de Rivera, se vio en la obligación de intervenir en más de una ocasión para calmar los ánimos. Así, envió diferentes circulares para aleccionar a sus militantes, valga ésta como ejemplo:

El objetivo principal será siempre, no la creación de profesionales del deporte, sino llegar a las mujeres en favor de una labor de convivencia social (salud, disciplina, coordinación) y formativa (emulación, camaradería y solidaridad), por medio del deporte, aprovechando las horas de ocio, pero sin perturbar el trabajo o el estudio (PRIMO DE RIVERA, 1953, documento 10).

En relación a ello cabe preguntarse: ¿la extensión de la Educación Física y el deporte dentro de la población femenina no traería aparejado el aumento del nivel deportivo y, como consecuencia, la aparición de deportistas cada vez mejores? Es cierto que habría que sopesar otros aspectos como la formación de los técnicos deportivos o la calidad y cantidad de materiales e instalaciones deportivas, pero indudablemente al aumentar el número de practicantes, crece no sólo el nivel de competencia dentro de cada deporte (puesto que al haber más contendientes, las exigencias deportivas aumentan), sino las posibilidades de encontrar a mujeres especialmente dotadas que descollen sobre el resto. Al respecto hay un dato muy esclarecedor, en 1940 el deporte era una práctica esporádica propia de las clases más privilegiadas, pero en 1970 más de 90.000 mujeres practicaban deporte y, por supuesto, competían en los campeonatos que desde el nivel municipal al nacional se organizaban en España y es más, las competiciones internacionales,como los Juegos Olímpicos, no les eran desconocidos, puesto que España tuvo participación femenina desde 1960 (ZAGALAZ, 2001). 
Por otro lado, este comportamiento dispar entre lo trazado por la Delegada Nacional y lo hecho por las afiliadas, plantea estas cuestiones: ¿Acaso las bases de la Sección Femenina estaban desafiando a su líder? ¿Era posible que la todopoderosa Pilar Primo de Rivera, que llegó a decir que la Sección Femenina y ella eran la misma cosa, no pudiera controlar a sus propias afiliadas? No, ni mucho menos, lo que ocurría es que las circunstancias le exigían un comportamiento ambivalente. Ella misma lo explicaba de la siguiente forma:

España vivía todavía unos criterios timoratos, y excesivamente pacatos que no hacían demasiado fácil nuestra labor...Es difícil hacerse una idea del escándalo que en algunas personas provocaba la aparición de aquella juventud faldicorta... compitiendo en los estadios deportivos. Las dirigentes tenían que buscar el equilibrio, avanzar sin comprometer nada esencial y hacerlo con prudencia, para evitar las críticas injustas(MARTíNEZ, 2008, p. 63).

De hecho, en relación al deporte de competición y la generación de deportistas de élite, y a pesar de lo expresado con anterioridad, Pilar Primo de Rivera hizo la siguiente alocución en el Primer Campeonato Nacional de Deportes de la Sección Femenina, allá por el año 1939:

Tiene que ganar vuestro equipo, no porque sea el vuestro, sino porque representáis a la Falange del sitio que sea, tenéis que ganar contra equipos internacionales cuando se presenten enfrente de vosotras, porque sois la Falange de España, tenéis que vencer en las Olimpíadas, porque el mundo entero se va a enterar de que gana la Falange.

Que vuestra ambición de deportistas esté siempre al servicio de la Falange. Adiestraos bien y preparaos con tenacidad, porque las cosas hechas a la ligera nunca dan buenos resultados, y además tenéis que saber que en la vida no se consigue nada por casualidad, vencen siempre los mejores (PRIMO DE RIVERA, 1942, p. 44).

Como se observa, no sólo abogaba por la competición deportiva sino que veía en estas prácticas un magnífico medio para fomentar unos valores que poco tenían que ver con la sumisión, la pasividad, la fragilidad o la abnegación, sino con el esfuerzo y la superación personal, dando un gran valor a ganar; incluso exhortaba a las deportistas a vencer en los Juegos Olímpicos.

Si los aspectos anteriores, referidos a la falta de permisividad con el deporte femenino y al intento de coacción del carácter agonístico de éste, fueron grandes escollos a superar, posiblemente no llegaron a ser tan problemáticos como el de la indumentaria deportiva. Por ejemplo el Arzobispo de Toledo afirmaba que "la vestimenta femenina no sólo debía llegar hasta las rodillas, pues un cuello descubierto es inmoral. Mangas que no cubren los codos deben ser consideradas inmorales también" (OFER, 2009, p. 117). Este contexto provocó que no fueran pocas las misivas de Pilar Primo de Rivera para, de nuevo, tratar de calmar los ánimos:

Uniforme deportivo obligatorio para participar en los V Campeonatos Nacionales de Gimnasia: pantalón bombacho azul marino cerrado por el lado izquierdo. Blusa blanca muy larga [...] En la prueba de rítmica se empleará como uniforme la túnica utilizada en la concentración de Medina, con pantalones bombachos debajo (PRIMO DE RIVERA, 1945, p. 6).

En los campeonatos de natación: No podrá participar ninguna camarada que no lleve el traje de baño reglamentario, que está dibujado en la circular n- 74; los albornoces blancos reglamentarios, en el recinto de la piscina estarán todas las participantes 
con el albornoz puesto. Sólo en el momento de tirarse al agua se lo quitarán, volviéndoselo a poner apenas salgan de ella (PRIMO DE RIVERA, 1945, p. 16).

Sin embargo, como ella misma explicaba en una entrevista que le realizaron en abril de 1986, no le quedó más remedio que aceptar las exigencias que en torno a la indumentaria deportiva marcaba el estamento eclesiástico. Si bien, con los años, fueron liberándose de las limitaciones impuestas al respecto y llegaron incluso a vestir maillots y pantalones cortos (PUJADAS et al., 2014). Pilar Primo de Rivera lo explicaba de la siguiente forma:

No, al principio, claro, nosotras mismas tuvimos que aceptar unos procedimientos un poco ñoños, por ejemplo los pololos ${ }^{4}$ y todo eso porque si no, no se hubiera admitido[...] a la sociedad española tal como estaba constituida le hubiera parecido un escándalo si salen las niñas como salieron después con sus maillots y sus cosas, pero no, no hubo ningún desquiciamiento en este sentido (MARTíNEZ, 2008, p. 73).

Siempre con un talante positivo y evitando el enfrentamiento directo, lograron no pocos avances en el campo de la Educación Física y el deporte femenino. Otro ejemplo claro al respecto fue la ya citada prohibición del atletismo entre las féminas, pues si bien la acataron, paralelamente introdujeron las carreras y los saltos (dos de las especialidades atléticas) en todos los programas de la asignatura de Educación Física. Esta extraña prohibición estuvo presente hasta 1961, cuando se permitió practicar atletismo a las mujeres, aunque éstas llevaban años practicándolo en las clases de Educación Física. De hecho, su práctica estaba tan extendida que en 1966, con motivo de la celebración en España de los VIII Juegos Internacionales Femeninos de la FISEC (Federación Internacional Deportiva de la Enseñanza Católica), organizados por la Sección Femenina y con la participación de ocho países europeos, el atletismo encabezó la lista de deportes, como se observa en el cartel publicado en Mundo Deportivo (JUEGOS..., 1966) en enero de ese mismo año.

Pero la Sección Femenina llegó a ir mucho más allá, utilizando la Educación Física y el deporte como medio de inserción de las mujeres en el reducido y dificultoso mundo laboral femenino. Ello lo consiguió a través del gran impulso que dio a la profesión de profesora de Educación Física. Ésta no era una ocupación desconocida para las mujeres, pues ya se formaron profesoras en la Escuela Central de Profesores y Profesoras de Gimnástica en el siglo XIX (ZORRILLA, 2002), pero es patente la promoción que hizo la Sección Femenina de esta profesión, pues no sólo situó a profesoras de Educación Física en los colegios de toda España, sino que incluso llegó a crear centros de formación al respecto.

En 1941 el Decreto de 2 de septiembre creó la Academia Nacional Isabel la Católica, que tenía como finalidad la formación de instructoras de la Sección Femenina. Años más tarde, la alta demanda de profesoras especialistas encontró respuesta en 1950 con la creación de otro centro de similares características, la Escuela de Instructoras Generales de las Navas del Marqués, inaugurada en 1951. Como sostiene López (2002), no sólo fueron éstos los centros de la Sección Femenina que capacitaban a sus alumnas para impartir Educación Física, también se abrieron otras escuelas, que convivieron con las anteriores, en ciudades como Málaga, Cádiz, Vitoria o Castellón.

Tras todo lo expuesto y a modo de síntesis, caben hacerse dos preguntas: ¿Por qué la Sección Femenina se empeñó con tanta tenacidad en que la Educación Física y el deporte

4 Los pololos eran faldas que cubrían hasta las rodillas, debajo tenían un pantalón que terminaba con un fuerte elástico ceñido a ambas rodillas. 
formaran parte de la vida de las mujeres de la época?, ¿por qué tanto esfuerzo en fomentar unas prácticas para las que, inicialmente, no tenían formación y que tenían una relevancia relativa dentro del modelo de mujer (hija, madre y esposa) que propugnaban?

Tal vez las respuestas se encuentren en discursos como éste de Pilar Primo de Rivera:

La timidez y la pasividad mostrada por algunas mujeres que ocupan altos cargos son a veces nada más que un patrón de comportamiento resultante del condicionamiento social [...]. No hay duda de que el mundo del deporte proporciona a las personas la fuerza para proteger a la esencia de su personalidad. Por tanto, vemos en las cualidades de liderazgo, iniciativa y toma de decisiones en el deporte requiere un canal perfecto para la promoción del papel de la mujer en la sociedad (PRIMO DE RIVERA, 1962, documento 45).

\section{CONCLUSIONES}

La Educación Física y el deporte femenino, si bien fueron definidos inicialmente dentro de los cánones del modelo de mujer (ama de casa, madre y esposa) que trató de imponerse, se utilizaron como medios de transgresión de dicho modelo. Con ello no se está planteando que sirvieran como forma de oposición a este arquetipo, porque la Sección Femenina creía en él y, de hecho, nunca se enfrentó de forma directa al resto de poderes del régimen en relación a reformular este ideal femenino, si bien, como se ha argumentado, a este modelo se le fueron añadiendo gradualmente nuevos roles de cariz progresista, sobre todo a partir de la década de 1960, pero siempre sin contradecir la esencia del mismo. En relación a estos cambios paulatinos, tanto la Educación Física como el deporte femenino contribuyeron activamente, abriendo nuevos horizontes que, inicialmente, le estaban vedados a las mujeres y que lentamente se fueron aceptando.

¿Cuáles fueron esas transgresiones del modelo femenino franquista? En primer lugar, una vez contenidas las reticencias que en torno a la Educación Física y el deporte femenino presentaron la Iglesia Católica y el sector político ultraconservador, las mujeres no sólo comenzaron a practicar deporte en base al cuidado de su salud, con una clara orientación hacia la maternidad y la política eugenésica propugnada, siempre respetando los principios morales impuestos y teniendo bien presente que era una forma de servir a su nación; sino que comenzaron a entrenar, a competir, a ganar y a perder. En definitiva, a disfrutar de la base agonística del deporte. Este no es un hecho baladí, puesto que la mujer debía ser sumisa y abnegada, siempre presta para el cuidado del hogar, del marido y los hijos. Sin embargo, con el fomento de la competición deportiva entre las féminas, se estaban transmitiendo una serie de valores (orgullo, competitividad, valor o esfuerzo) que nada tenían que ver con el prototipo de mujer que se trataba de imponer desde los estamentos rectores del franquismo.

En segundo lugar, hay que destacar las argucias que en más de una ocasión puso en funcionamiento la Sección Femenina para no sólo justificar la necesidad de la Educación Física y el deporte entre la mujeres, sino incluso para practicar deportes que les estaban vedados. Ejemplo paradigmático al respecto es el atletismo, prohibido hasta 1961, pero cuyas especialidades de carreras y saltos estaban incluidas en todos los programas formativos de la Educción Física femenina.

En tercer lugar, siendo posiblemente la transgresión más llamativa por el componente sexual que conllevaba, está la evolución de la indumentaria deportiva: de los pololos (que 
por su estructura excluían la posibilidad de que quedara al descubierto cualquier parte del cuerpo femenino por encima de las rodillas) a los ajustados maillots que incluso dejaban al descubierto las piernas de las mujeres. Bien es cierto que esta evolución se produjo a partir del Desarrollismo, pero ésta fue otra de las características de la Sección Femenina: aprovechar cualquier impulso externo para lograr beneficios en relación a introducir matices progresistas en el modelo de mujer impuesto.

En cuarto y último lugar, hay que destacar que la Educación Física y el deporte permitieron ampliar el campo laboral de las mujeres, pues a partir de su desarrollo se dio un gran impulso a la profesión de profesora de Educación Física. Una labor que, obviamente, superaba, a nivel educativo y laboral, el papel queen la España franquista, sobre todo durante las primeras décadas, tuvieron asignado las mujeres.

Por tanto, no fueron pocas las disonancias entre el modelo de mujer que se estaba tratando de imponer y los valores y roles que se transmitían a las mujeres desde la Educación Física y el deporte. Tal vez lo más llamativo de todo ello sea la posición paradójica de la Sección Femenina, pues fue la principal impulsora de estas prácticas e introdujo valores progresistas a partir de ellas, aunque esta organización siempre defendió el modelo femenino impuesto y evitó enfrentarse en relación a ello con el resto de facciones de la cúpula de poder franquista.

\section{REFERENCIAS}

ALTED, Alicia. Las mujeres en la sociedad española de los años cuarenta. In: Las mujeres y la Guerra Civil Española.Madrid: Ministerio de Cultura, Instituto de la Mujer, 1991. p. 293-303.

ÁLVAREZ, Raquel. Eugenesia y fascismo en la España de los años treinta. In: HUERTAS, Rafael; ORTIZ, Carmen (Eds). Ciencia y Fascismo. Madrid: Doce Calles, 1997. p. 77-96.

EL ATLETISMO femenino. Correo de Mallorca, p.28, 4 nov. 1941.

BARRACHINA, Mํㅡㄹ Aliñe. Ideal de la Mujer Falangista. Ideal Falangista de la Mujer. In: LAS MUJERES y la Guerra Civil Española. Madrid: Ministerio de Cultura. Instituto de la Mujer, 1991. p. 211-217.

CAMPOS, Ricardo. Autoritarismo y eugenesia punitiva: higiene racial y nacionalcatolicismo en el franquismo, 1936-1945. História, Ciências, Saúde-Manguinhos, n. 23, supl., p. 131-147, dez.2016.

CAMPOS, Ricardo; HUERTAS, Rafael. Medicina mental y eugenesia: los fundamentos ideológicos de la psiquiatría franquista en la obra de Antonio Vallejo-Nájera. Historia del presente, n. 20, p. 11-21, 2012.

CHAVES, Rafael.Manual Escolar de Educación Física. Madrid: Doncel, 1960.

DE LA GRANDA, Antonio. Los fundamentos biológicos del trabajo en la mujer. Revista de Trabajo 2a época, n. 5, p. 489-498, 1947.

ECONOMÍA doméstica para bachillerato y magisterio. Madrid: Sección Femenina, 1958. 
ESPAÑA. Decreto de 19 de abril de 1937 de Unificación. Boletín Oficial del Estado, Madrid, 20 abr. 1937. Disponible en: <https://www.boe.es/datos/pdfs/BOE//1937/182/A01033-01034.pdf>. Acceso en: 30 ago. 2017.

ESPAÑA. Decreto de 2 septiembre de 1941. Boletín Oficial del Estado, Madrid, 8 sept. 1941. Disponible en: <https://www.boe.es/datos/pdfs/BOE//1941/251/A06886-06887.pdf>. Acceso en: 30 ago. 2017.

ESPAÑA. Decreto de 28 de diciembre de 1939. Boletín Oficial del Estado, Madrid, 29 dic.1939. Disponible en:<https://www.boe.es/datos/pdfs/BOE//1939/363/A07347-07348.pdf > . Acceso en: 30 ago. 2017.

ESPAÑA. Ley de 6 de diciembre de 1940. Boletín Oficial del Estado, Madrid, 7 dic. 1940. Disponible en:<http://www.boe.es/datos/pdfs/BOE/1940/342/A08392-08394.pdf>. Acceso en: 30 ago. 2017.

ESPAÑA. Ley 56/1961 de 22 de julio de 1961. Boletín Oficial del Estado, Madrid, 24jul.1961. Disponibleen:<https://www.boe.es/buscar/doc.php?id=BOE-A-1961-14132> Acceso en: 30 ago. 2017.

FLICK, Uwe. Introducción a la investigación cualitativa. Madrid: Morata, 2007.

HERRERO, Heliodoro. Por la educación hacia la revolución: La contribución de la Educación Física a la construcción del imaginario social del franquismo. Revista Internacional de Medicina y Ciencias de la Actividad Física y el Deporte, n. 4, p. 21-36, 2002.

LEÓN, Fray Luis de. La perfecta casada. Madrid: Taurus, [1583] 1987.

LÓPEZ, Iván. Evolución histórica de la formación inicial del profesorado de Educación Física en España. Revista Fuentes, n. 4, p.164-187, 2002.

MANRIQUE, Juan Carlos. La Educación Física Femenina y el ideal de mujer en la etapa franquista. Revista Internacional de Medicina y Ciencias de la Actividad Física y el deporte, n. 10, p. 83-100, 2003.

MARSHALL, Catherine; ROSSMAN, Gretchen. Designing qualitative research. Los Angeles: SAGE, 2011.

MARTíNEZ, Ascensión. Entrevista a Pilar Primo de Rivera. Abril de 1986. In: Voces del pasado: la élite cultural de España. Madrid: UNED, 2008. p. 47-78.

MIRANDA, María de. Franco y el deporte femenino. Fondo de la Asociación "Nueva andadura", Serie Azul, Carpeta 42, documento 52, 1942.

MIRANDA, María de. Sobre la necesidad de la Educación Física. Fondo de la Asociación "Nueva andadura", Serie Azul, Carpeta 42, documento 49, 1940.

MÒDOL, Jarné. Models formals i sentimentals al Server de la fenineïtat : la postguerra a Lleida (1939-1945).llerda, n.49, p. 189-207, 1991.

MOLINERO, Carme. Mujer, franquismo, fascismo. La clausura forzada en un "mundo pequeño". Historia Social, n. 30, p. 97-117, 1998.

NUÑEZ, Mirta. Tríptico de mujeres de posguerra: de la mujer comprometida a la marginal. Historia del presente, n. 4, p. 47-60, 2004. 
OFER, Inbal.Señoritas in blue. The making of a female political elite in Franco's Spain. Great Britain: Sussex Academic, 2009.

PASTOR, Mํㅡㄹ Inmaculada. La educación femenina en la postguerra (1939-1945). El caso de Mallorca. Madrid: Ministerio de Cultura. Instituto de la Mujer, 1984.

PIO XII, Papa.Alocución a las mujeres de las asociaciones cristianas de Italia el 21 de octubre de 1945. In:Colección de Encíclicas y Documentos Pontificios. Madrid: Junta Nacional de ACE, 1967. v.1, p. 1688-1689.

PRESTON, Paul. Franco: caudillo de España. Madrid: Debate, 2015.

PRIMO DE RIVERA, Pilar. Circular a las Delegadas provinciales de 19 de agosto.Fondo de la Asociación "Nueva andadura", Serie Roja, Carpeta 84, documento 10, 1953.

PRIMO DE RIVERA, Pilar. Circular número 235. Boletín Oficial de la Delegación Nacional de Deportes, Madrid, n. 23, 1945, p. 16.

PRIMO DE RIVERA, Pilar. Circular número 255. Boletín Oficial de la Delegación Nacional de Deportes, Madrid, n. 27, 1945, p. 6.

PRIMO DE RIVERA, Pilar. Discurso. Fondo de la Asociación "Nueva andadura”, Serie Azul, Carpeta 118, documento 45, 1962.

PRIMO DE RIVERA, Pilar. Discursos, Circulares y Escritos. Madrid: Falange Española y Tradicionalista: Gráficas Afrodisio Aguado, 1942.

PRIMO DE RIVERA, Pilar. La Sección Femenina. Historia y Organización. Madrid: Falange Española y Tradicionalista, 1952.

PRIMO DE RIVERA, Pilar. Recuerdos de una vida. Madrid: Editorial Dyrsa, 1983.

PUJADAS, Xavier et al.Sports, morality and body: the voices of sportswomen under Franco's dictatorship. International Review for the Sociology of Sport,v. 51, n. 6, p. 679-698, 2016.

RAMÍREZ-MACÍAS, Gonzalo. Women archetype and Female Physical Education during Franco's Dictatorship. The International Journal of the History of Sport, v. 29, n. 11, p. 15131528, 2012.

SCANLON, Geraldine. La mujer bajo el franquismo. Tiempo de Historia, n. 27, p. 4-28, 1977.

JUEGOS INTERNACIONALES FEMENINOS DE LA FISEC, 8, 1966,Madrid.Mundo deportivo. Madrid:Federación Internacional de la Enseñanza Católica, 1966.

ZAGALAZ, Mํㅡㄴ Luisa. La Educación Física femenina durante el franquismo:la Sección Femenina. Apunts, Educación física y deportes, n. 65, p. 6-16, 2001.

ZORRILLA, Pedro Pablo Sanz. La Escuela Central de Profesores y Profesoras de Gimnástica (1887-1892). Apunts, Educación física y deportes, n.69, p. 6-16, 2002. 
